\title{
Two-Photon Luminescence Imaging of Cancer Cells using Molecularly Targeted Gold Nanorods
}

\author{
Nicholas J. Durr $\dagger^{\prime}$, Timothy Larson ${ }_{\star \neq}$ Danielle K. Smith ${ }^{\ddagger}$, Brian A. Korgel ${ }^{\ddagger}$, Konstantin \\ Sokolov ${ }^{\dagger},{ }^{*}$, and Adela Ben-Yakart, ${ }^{* *}$ \\ † Department of Biomedical Engineering; The University of Texas at Austin; Austin, TX 78712 \\ ‡ Department of Chemical Engineering; Texas Materials Institute, Center for Nano- and Molecular \\ Science and Technology; University of Texas at Austin; Austin TX 78712 \\ * Department of Biomedical Engineering; The University of Texas M.D. Anderson Cancer Center; \\ Houston, TX 77030
}

\begin{abstract}
We demonstrate the use of gold nanorods as bright contrast agents for two-photon luminescence (TPL) imaging of cancer cells in a three dimensional tissue phantom down to 75 $\mu \mathrm{m}$ deep. The TPL intensity from gold nanorod labeled cancer cells is three orders of magnitude brighter than the two-photon autofluorescence (TPAF) emission intensity from unlabeled cancer cells at $760 \mathrm{~nm}$ excitation light. Their strong signal, resistance to photobleaching, chemical stability, ease of synthesis, simplicity of conjugation chemistry, and biocompatibility make gold nanorods an attractive contrast agent for two-photon imaging of epithelial cancer.
\end{abstract}

More than $85 \%$ of all cancers begin as precancerous lesions that are confined to the surface epithelium, which can be as thick as $500 \mu \mathrm{m}$ in human tissue. Two-photon imaging (TPI) is a powerful technique for the early diagnosis of epithelial cancers because it permits non-invasive imaging of sub-cellular features potentially hundreds of micrometers deep into tissue. ${ }^{1-3} \mathrm{~A}$ recent study has demonstrated that morphological and fluorescence quantification from TPI of endogenous fluorophores can be used to distinguish cancerous and precancerous from normal tissue down to $40 \mu \mathrm{m}$ deep. ${ }^{4}$ By attaching a fluorescent contrast agent to a non-fluorescent target of interest, TPI also has the ability to monitor a variety of additional biomolecular signatures that are more strongly indicative of cancer. The promise of this technique has prompted researchers to investigate the use of traditional and engineered organic fluorophores, 5 as well as new classes of luminescent contrast agents such as quantum dots ${ }^{6}$ and metallic nanoparticles. ${ }^{7-10}$ Though fluorescent semiconductor quantum dots offer much larger twophoton action cross sections than organic fluorophores (>10,000 GM compared to 1-300 GM for organic fluorophores), ${ }^{11}$ the most well-studied quantum dots have heavy metals that are cytotoxic, making them unsuitable for in vivo clinical applications. Gold nanoparticles, on the other hand, are biocompatible ${ }^{12}$ and can have large two-photon action cross sections (larger than 2,000 GM for gold nanorods). ${ }^{9}$

Single-photon induced luminescence was first reported from bulk copper and gold by Mooradian in $1969 .{ }^{13}$ Later, Boyd et. al. found that roughened metal surfaces exhibited much higher single-photon induced luminescence efficiency than smooth surfaces, and furthermore

\footnotetext{
**Corresponding Author. Email: ben-yakar@mail.utexas.edu. Phone (+1) 512-475-9280. Mechanical Engineering Department; The University of Texas at Austin; 1 University Station C2200; Austin, TX 78712.

Supporting Information Available: Emission spectra of nanorods and control images overlapped with white light transmission images as well as low power TPAF control images are included as supporting information. This material is available free of charge via the Internet at http://pubs.acs.org.
} 
induced observable two-photon induced luminescence, which depends quadratically on excitation intensity. ${ }^{14}$ The luminescence enhancement from roughened surfaces is likely a result of efficient resonant coupling of specific frequencies of light to surface plasmons. Twophoton luminescence (TPL) has been identified as a serial process involving sequential absorption of photons and emission from the recombination of electrons in the sp-band and holes in the d-band. ${ }^{15}$ This absorption process in TPL is different than two-photon excitation in fluorophores, which requires near simultaneous absorption of two coherent photons.

Gold nanorods exhibit highly efficient single- and two-photon induced luminescence, ${ }^{9}, 16$ which may be due to their ability to sustain resonating surface plasmons with minimal damping. ${ }^{17}$ Furthermore, their longitudinal plasmonic resonance can be tuned to the near infrared wavelengths, where biological tissue exhibit relatively small extinction coefficients. The TPL emission spectra shows that much of the emission light is also in the NIR region. ${ }^{9}$ These optical properties make gold nanorods an attractive contrast agent for biomedical imaging of highly scattered tissue. With the use of near-field scanning microscopy and the ability to synthesize metallic particles in a variety of shapes, progress is being made in understanding the optical properties of gold nanorods. ${ }^{15,18}$ However, relatively few efforts have explored the applications of gold nanorods as contrast agents for imaging, ${ }^{8,9,19}$ and no reports have been found that use gold nanorods for molecularly specific nonlinear imaging.

We present TPL images of gold nanorod-labeled cancer cells acquired up to $75 \mu \mathrm{m}$ deep in a tissue phantom. By embedding cells in a collagen matrix, the performance of nanorods as contrast agents for deep-tissue imaging was explored. We found that TPL imaging of labeled cancer cells could be performed using less than 60 times the laser excitation power needed for two-photon autofluorescence (TPAF) imaging from unlabeled cancer cells, corresponding to a three order-of-magnitude increase in emitted signal for equal excitation intensity.

Imaging was performed on a home-built inverted multiphoton microscope shown in Figure 1. A femtosecond Ti:Sapphire laser (Mai Tai, Spectra Physics, Newport) was used as the excitation source. The pulse length at the sample at $760 \mathrm{~nm}$ wavelength was estimated to be 250 fs using a two-photon absorption collinear autocorrelator with a photodiode (G1117, Hamamatsu) and an objective lens group delay dispersion value of $3520 \mathrm{fs}^{2} .20$ The laser was raster scanned into the back aperture of an oil-immersion objective lens (NA=1.4 64x, Zeiss) with a set of galvanometric scanning mirrors $(6215 \mathrm{H}$, Cambridge Technologies). Emitted light was epi-collected, reflected by a cold mirror (400-700 nm reflectance, HT-1.00, CVI Laser), passed thorough a laser filter (BG-38, Schott, blocks $>700 \mathrm{~nm}$ ), detected with a cooled GaAsP photomultiplier tube (H7422-40, Hamamatsu), and assembled into an image in real-time with a data acquisition card (6111E, National Instruments). Given the response of the PMT and optics used, the system collected emission light between $400 \mathrm{~nm}$ and $700 \mathrm{~nm}$. A field of view of $145 \mu \mathrm{m}$ was scanned into a $512 \times 512$ pixel image at a rate of 1.5 frames per second. The sample was moved in the axial direction with a piezoelectric actuator (P280, Physik Instrumente).

Gold nanorods were synthesized using a seed-mediated, surfactant-assisted growth method in a two step procedure. ${ }^{21-23}$ Colloidal gold seeds ( $~ 1.5 \mathrm{~nm}$ diameter $)$ are first prepared by mixing aqueous solutions of hexadecylcetyltrimethylammonium bromide (CTAB, $0.1 \mathrm{M}, 9.75 \mathrm{~mL}$ ) and hydrogen tetrachloroaurate(III) hydrate $(0.01 \mathrm{M}, 250 \mu \mathrm{L})$. (Note: For nanorods to form, we found it necessary to use low purity CTAB from Fluka (96\% purity), as nanorods did not form when high purity CTAB ( $\geq 99 \%$ purity) was used. $)^{24}$ The colloidal gold seeds are then injected into an aqueous "growth solution" of CTAB $(0.1 \mathrm{M}, 9.5 \mathrm{ml})$, silver nitrate $(0.01 \mathrm{M}$, varying amounts of silver between $20-120 \mu \mathrm{L}$ depending on desired nanorod aspect ratio), hydrogen tetrachloroaurate(III) hydrate $(0.01 \mathrm{M}, 500 \mu \mathrm{L})$, and ascorbic acid $(0.1 \mathrm{M}, 55 \mu \mathrm{L})$. An aqueous solution of sodium borohydride $(.01 \mathrm{M}, 600 \mu \mathrm{L})$ is then added. ${ }^{25,} 26$ Nanorods 
were purified by several cycles of suspending them in ultrapure water followed by centrifugation. The nanorods are isolated in the precipitate and excess CTAB is removed in the supernatant. Figure 2a shows a transmission electron microscopy (TEM) image of synthesized gold nanorods that have an average aspect ratio of $3.4 \pm 0.6$. Figure $2 b$ shows that the longitudinal plasmon mode of these nanorods is centered at $754 \mathrm{~nm}$.

To functionalize the gold nanorods, the positive surface potential was converted to a negative surface potential by coating the CTAB with polystyrene sulfonate (PSS). ${ }^{27} \mathrm{PSS}$ (MW $14 \mathrm{kD}$, $10 \mathrm{mg} / \mathrm{mL}$ in $1 \mathrm{mM} \mathrm{NaCl}$ solution) was added to the nanorod suspension in a 1:10 volume ratio and allowed to react for 30 minutes. The particles were then collected via centrifugation at $2000 \mathrm{~g}$ for 30 minutes, resuspended in $\mathrm{NaCl}(1 \mathrm{mM})$ and reacted with another aliquot of PSS solution. Following the second PSS incubation the particles were washed twice in water and then resuspended in HEPES (40 mM, pH 7.4) for compatibility with the antibody solution. Anti-EGFR antibody (clone 29.1, Sigma) was purified using Centricon $100 \mathrm{kD}$ MWCO filters, then resuspended in HEPES ( $40 \mathrm{mM}, \mathrm{pH} 7.4,200 \mu \mathrm{g} / \mathrm{mL}$ ). For the control, a non-specific antibody was used in place of the anti-EGFR antibody (clone MOPC 21, Sigma). Antibody solution and nanorods were mixed at 1:1 volume ratio and allowed to interact for 45 minutes. PEG (MW $15 \mathrm{kD}, 10 \mathrm{mg} / \mathrm{mL}$ in 1x PBS) was then added for stability and the particles were centrifuged to remove unbound antibodies.

Three-dimensional tissue phantoms were prepared using EGFR-overexpressing A431 skin cancer cells. The cells were purchased from American Type Culture Collection and cultured in DMEM supplemented with 5\% FBS. Cells were harvested via trypsinization and resuspended in $1 \mathrm{x}$ PBS at a concentration of $6^{*} 10^{6}$ cells $/ \mathrm{mL}$ as determined with a hemacytometer. The cell suspension was mixed with either EGFR targeted or non-specifically targeted nanorods in a 1:1 volume ratio and allowed to interact for 45 minutes. The cells were then spun down at $200 \mathrm{~g}$ for five minutes to remove unbound particles and the cells were resuspended in a buffered collagen solution at a concentration of $7.5^{*} 10^{7}$ cells $/ \mathrm{mL}$. The collagen/cell mix was pipetted into a 120 um spacer (Molecular Probes) and sealed with a coverslip for imaging.

Two-photon induced luminescence from the gold nanorods was verified by measuring the dependence of the emission intensity on the excitation power. Gold nanorods were sparsely deposited on a glass coverslip and single emission spots were examined. A quadratic dependence of $2.05 \pm 0.06$ on the incident power was observed for increasing excitation powers from $30 \mu \mathrm{W}$ to $90 \mu \mathrm{W}$, indicating that the excitation is a two-photon process (Figure $2 \mathrm{c}$ ). The TPL signal also exhibited $\cos ^{4}$ dependence on excitation polarization, which has been observed in other reports. ${ }^{9}$ When a droplet of PBS was placed on the nanorod-coated coverslip, emission from nanorods that maintained their attachment to the coverslip was unchanged in both magnitude and the quadratic dependence of emission intensity. The point spread function (PSF) of the TPL imaging setup was obtained by recording the intensities of a small spots that exhibited strong polarization sensitivity. From the full-width at half maximum of the PSF, the radial and axial resolutions were $320 \mathrm{~nm}$ and $625 \mathrm{~nm}$, respectively. A similar resolution was also measured from the PSF of $100 \mathrm{~nm}$ diameter fluorescent beads.

Figure 3 presents two-photon images of a single layer of labeled and unlabeled cells at $760 \mathrm{~nm}$ excitation wavelength. The images show successful labeling, cellular distribution of EGFR, and relative brightness of nanorods. The unlabeled cells (Figure 3a) show a relatively uniform distribution of TPAF signal throughout cellular cytoplasm. No signal is associated with the nuclei, which do not have significant concentrations of endogenous fluorophores that can be excited in the visible region. In labeled cells (Figure 3b) bright rings can be easily seen; this is a characteristic pattern of EGFR labeling and has been also reported in confocal reflectance imaging of EGFR using spherical gold nanoparticles. ${ }^{28}$ The discrete bright spots in the 
cytoplasm of nanorod labeled cells are indicative of endosomal uptake of EGF receptors labeled with nanorods inside cells. The endosomal recycling of EGFR molecules is a normal biological function of living cancer cells. ${ }^{29,30}$ TPL images of cells treated with non-specifically conjugated nanorods (Figure 3c) show agglomeration of contrast agent and little attachment to cell membranes (see Figure S1 in supplementary information for overlapped TPL and whitelight transmission images). Using the same excitation power of $140 \mu \mathrm{W}$, the two-photon imaging of unlabeled cells gave a pure background noise black image (see Figure S2 in supporting information).

By tuning the wavelength from $710 \mathrm{~nm}$ to $910 \mathrm{~nm}$, it was found that $760 \mathrm{~nm}$ excitation wavelength yielded the brightest TPL signal from the nanorods, as well as the brightest TPAF signal from the cancer cells. This wavelength corresponds to the longitudinal plasmon resonance frequency of the nanorods. For the cancer cells, this finding is consistent with other reports that show that the biological molecules that are primarily responsible for the signal in TPAF imaging (NADH and flavins), have two-photon cross sections that increase with decreasing excitation wavelength from $1,000 \mathrm{~nm}$ to $750 \mathrm{~nm}$, and level off around $750 \mathrm{~nm} .^{3}$ Similarity of the optimal excitation wavelengths for both the gold nanorods and cellular autofluorescence provides a fair comparison of emission intensities between the two imaging modalities without the need for detector response and filter normalization. We found that TPL imaging of nanorod labeled cells required 64 times less power than TPAF imaging of unlabeled cells in order to achieve the similar collected intensity. Given the quadratic dependence of emission intensity on the incident power, this observation implies that for equal excitation powers, TPL imaging of nanorod labeled cancer cells can generate more than 4,000 times larger emission signal than TPAF imaging of unlabeled cells.

To test the imaging ability of TPL deep into tissue, we imaged cancer cells embedded in a collagen matrix that mimics the epithelium tissue. Figure 4 compares TPL with TPAF images of cancer cells obtained at different depths. In order to avoid delivery of large powers at deep levels, we increased our PMT gain and reduced imaging power compared to the configuration in single cell layer imaging. The high gain allowed imaging at ten times less power than the single layer cell experiments. To maintain a constant detected intensity throughout the phantoms, a $26 \%$ power increase was required for each $20 \mu \mathrm{m}$ increase in imaging depth. For TPAF and TPL imaging, this corresponded to increasing the power from $0.9 \mathrm{~mW}$ to $1.8 \mathrm{~mW}$ and from $35 \mu \mathrm{W}$ to $70 \mu \mathrm{W}$, respectively. The excitation power ratio of 26 between two imaging modalities indicates 675 times brighter TPL than TPAF. The difference in brightness between nanorod labeled cells in Figure 3 and 4 is likely related to the density of nanorods per cell and how the nanorods are distributed over the cell membrane. We found that we could image up to the working distance of the lens in both phantoms, but images deeper than $70 \mu \mathrm{m}$ were severely degraded by spherical aberrations due to the refractive index mismatch between the immersion oil and the aqueous sample. Remarkably, both TPL and TPAF image required the same increase in the excitation power at increasing imaging depths in order to maintain a constant emission intensity, indicating that even though nanorods are typically considered very strong scatterers of light, the bulk extinction coefficient was not appreciably increased in nanorods labeled phantoms.

We have demonstrated the effective use of gold nanorods as bright contrast agents for TPL imaging. By labeling cancer cells with anti-EGFR nanorod conjugates, molecular specific imaging could be carried out with very high signal-to-noise ratios deep into a tissue phantom. In cases where imaging depths will be limited by the maximum available power that can be delivered to the imaging plane without causing damage to tissue, gold nanorods might provide sufficient brightness to extend the maximum depth of imaging. Additionally, we have shown that the use of gold nanorods can expand the capabilities of TPI to allow non-invasive threedimensional imaging of a variety of new molecular signatures. 


\section{Supplementary Material}

Refer to Web version on PubMed Central for supplementary material.

\section{Acknowledgments}

We acknowledge Quoc Nguyen for providing the source code for his MPScan program, and Pengyuan Chen and Benny Hwang for their assistance in modifying the program for use in our microscope. Special thanks to Newport for their valuable support and technical assistance. D.S. and B.K. acknowledge partial funding of this research by The Robert A. Welch Foundation, the National Institutes of Health and the National Science Foundation (CHE-9876674). A.B. and N.D. acknowledge support by the National Institute of Health NIH Grant RO3 CA125774-01 and National Science Foundation Grants BES-0548673 and BES-0508266 (AB). N.D. is partially supported by a National Science Foundation IGERT Fellowship.

\section{References}

1. Konig K, Riemann I. J Biomed Opt 2003;8(3):432-9. [PubMed: 12880349]

2. Masters BR, So PT, Gratton E. Ann N Y Acad Sci 1998;838:58-67. [PubMed: 9511795]

3. Zipfel WR, Williams RM, Christie R, Nikitin AY, Hyman BT, Webb WW. Proc Natl Acad Sci U S A 2003;100(12):7075-80. [PubMed: 12756303]

4. Skala MC, Squirrell JM, Vrotsos KM, Eickhoff JC, Gendron-Fitzpatrick A, Eliceiri KW, Ramanujam N. Cancer Res 2005;65(4):1180-6. [PubMed: 15735001]

5. Albota M, Beljonne D, Bredas JL, Ehrlich JE, Fu JY, Heikal AA, Hess SE, Kogej T, Levin MD, Marder SR, McCord-Maughon D, Perry JW, Rockel H, Rumi M, Subramaniam G, Webb WW, Wu XL, Xu C. Science 1998;281(5383):1653-6. [PubMed: 9733507]

6. Larson DR, Zipfel WR, Williams RM, Clark SW, Bruchez MP, Wise FW, Webb WW. Science 2003;300(5624):1434-6. [PubMed: 12775841]

7. Farrer RA, Butterfield FL, Chen VW, Fourkas JT. Nano Lett 2005;5(6):1139-42. [PubMed: 15943457]

8. Sonnichsen C, Alivisatos AP. Nano Lett 2005;5(2):301-4. [PubMed: 15794615]

9. Wang H, Huff TB, Zweifel DA, He W, Low PS, Wei A, Cheng JX. Proc Natl Acad Sci U S A 2005;102 (44):15752-6. [PubMed: 16239346]

10. Yelin D, Oron D, Thiberge S, Moses E, Silberberg Y. Optics Express 2003;11(12):1385-1391. [PubMed: 19466009]

11. Zipfel WR, Williams RM, Webb WW. Nat Biotechnol 2003;21(11):1369-77. [PubMed: 14595365]

12. Abrams MJ, Murrer BA. Science 1993;261(5122):725-730. [PubMed: 8102010]

13. Mooradian A. Phys Rev Lett 1969;22:185-187.

14. Boyd GT, Yu ZH, Shen YR. Physical Review B Condensed Matter 1986;33(12):7923-7936.

15. Imura K, Nagahara T, Okamoto H. Journal of Physical Chemistry B 2005;109(27):13214-13220.

16. Mohamed MB, Volkov V, Link S, El-Sayed MA. Chemical Physics Letters 2000;317(6):517-523.

17. Sonnichsen C, Franzl T, Wilk T, von Plessen G, Feldmann J, Wilson O, Mulvaney P. Phys Rev Lett 2002;88(7):077402. [PubMed: 11863939]

18. Bouhelier A, Beversluis MR, Novotny L. Applied Physics Letters 2003;83(24):5041-5043.

19. Huang X, El-Sayed IH, Qian W, El-Sayed MA. J Am Chem Soc 2006;128(6):2115-20. [PubMed: 16464114]

20. Guild JB, Xu C, Webb WW. Applied Optics 1997;36(1):397-401. [PubMed: 18250687]

21. Gou LF, Murphy CJ. Chemistry of Materials 2005;17(14):3668-3672.

22. Jana NR, Gearheart L, Murphy CJ. Journal of Physical Chemistry B 2001;105(19):4065-4067.

23. Nikoobakht B, El-Sayed MA. Chemistry of Materials 2003;15(10):1957-1962.

24. The CTAB source was found to be critical for success in the gold nanorod synthesis. For nanorods to form using the published procedures, we found it necessary to use lower purity CTAB:CTAB from both Fluka (96\% purity) and MP Biomedical (98\% purity) gave Au nanorods. We could not make Au nanorods using CTAB obtained from the following manufacturers: Acros (99\% purity), Aldrich ( $\sim 100 \%$ purity), and Sigma (99\% purity). We attribute this difference to the purity in the CTAB.

However, we currently do not know what the impurity is or why it is important, only that it 
dramatically affects the product and the only way we could reproduce gold nanorod syntheses reported in the literature was to use the CTAB of lower purity from either Fluka or MP Biomedical. 25. Liu MZ, Guyot-Sionnest P. Journal of Physical Chemistry B 2005;109(47):22192-22200.

26. Murphy CJ, San TK, Gole AM, Orendorff CJ, Gao JX, Gou L, Hunyadi SE, Li T. Journal of Physical Chemistry B 2005;109(29):13857-13870.

27. El-Sayed IH, Huang X, El-Sayed MA. Nano Lett 2005;5(5):829-34. [PubMed: 15884879]

28. Sokolov K, Follen M, Aaron J, Pavlova I, Malpica A, Lotan R, Richards-Kortum R. Cancer Research 2003;63(9):1999-2004. [PubMed: 12727808]

29. Barnes, CJK.; Rakesh. Biology of the epidermal growth factor receptor family. Vol. 119. Springer; US: 2004

30. Schlessinger J. Cell 2000;103(2):211-225. [PubMed: 11057895] 


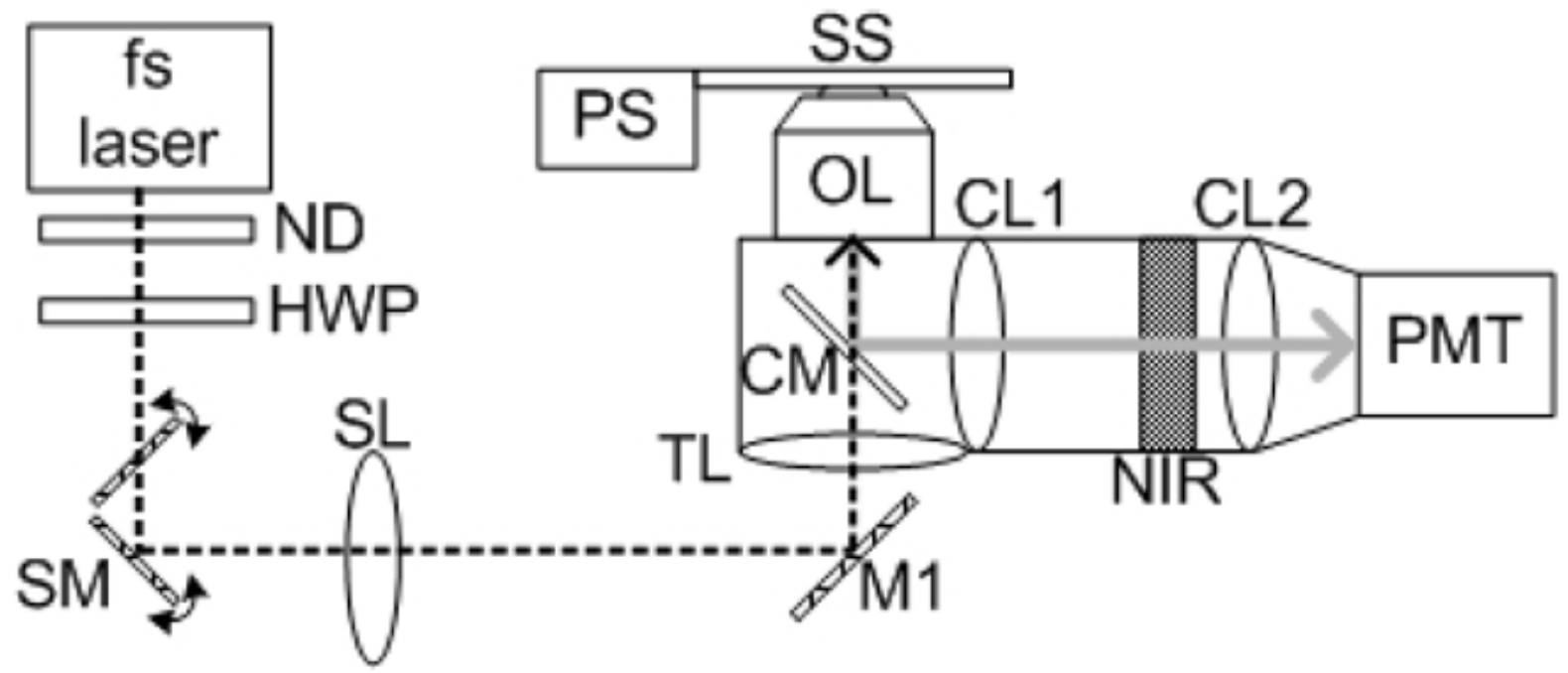

ND: Neutral Density Filters OL: Objective Lens HWP: Half Wave Plate SS: Sample Stage SM: Scanning Mirrors SL: Scan Lens M1: NIR mirror

TL: Tube Lens PS: Piezoelectric Stage CM: Cold Mirror CL1: Collecting Lens 1 NIR: Near-infrared filter CL2: Collecting Lens 2 PMT: Photomultiplier Tube

Figure 1.

Schematic of a home built two-photon microscope. System directs the laser pulses into a raster pattern using galvanometric scanning mirrors (SM). Lateral and axial resolutions of $320 \mathrm{~nm}$ and $625 \mathrm{~nm}$ are achieved with a 64x/1.4NA oil immersion objective lens (OL). 

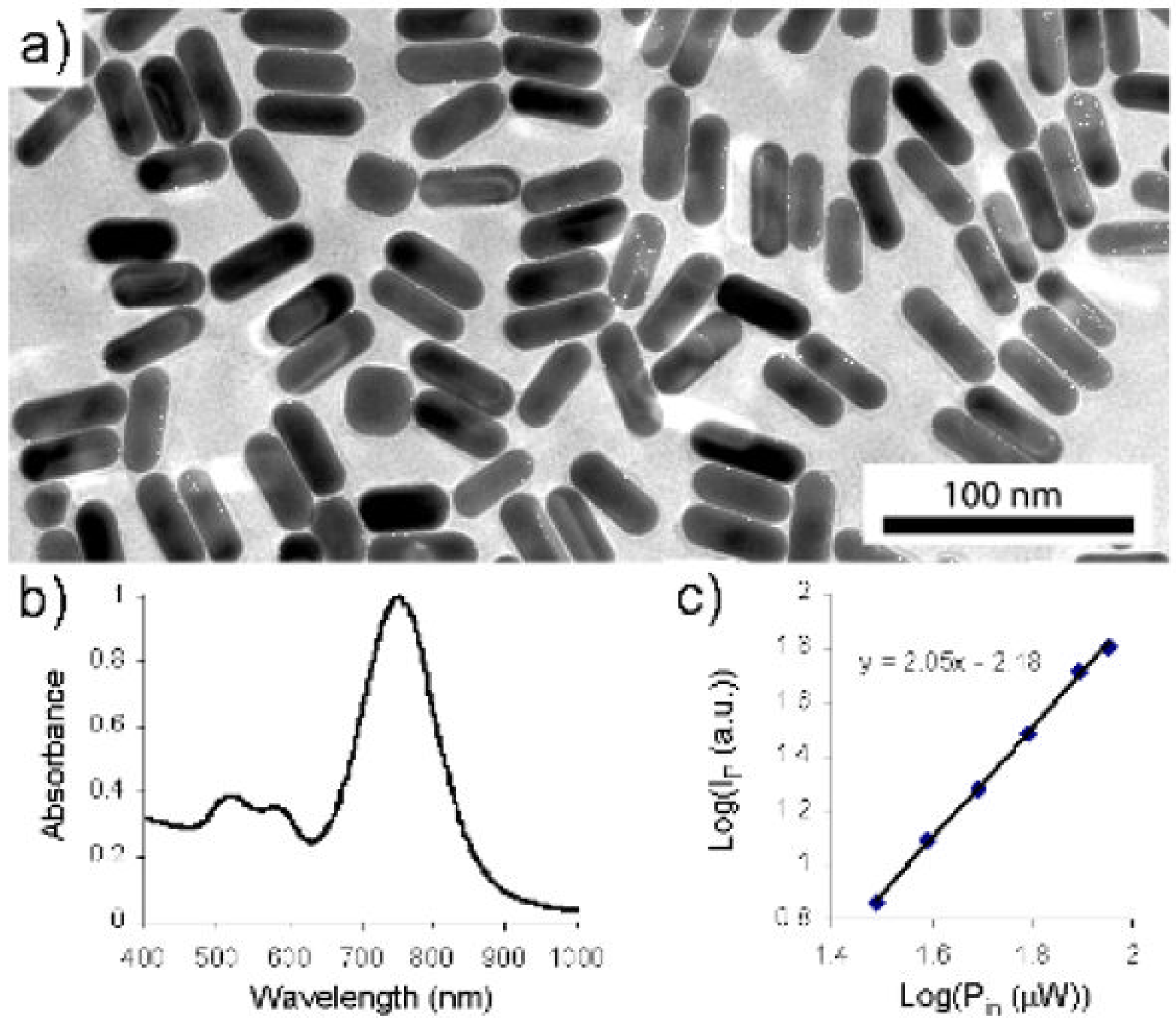

Figure 2.

Properties of the gold nanorods used as contrast agents. (a) TEM image of gold nanorods indicates an average length and width of $48.1 \pm 5.5 \mathrm{~nm}$ and $14.3 \pm 2.2 \mathrm{~nm}$, respectively. (b) Gold nanorod absorbance in aqueous solution. (c) Emission intensity dependence of gold nanorods on excitation power at $760 \mathrm{~nm}$. 


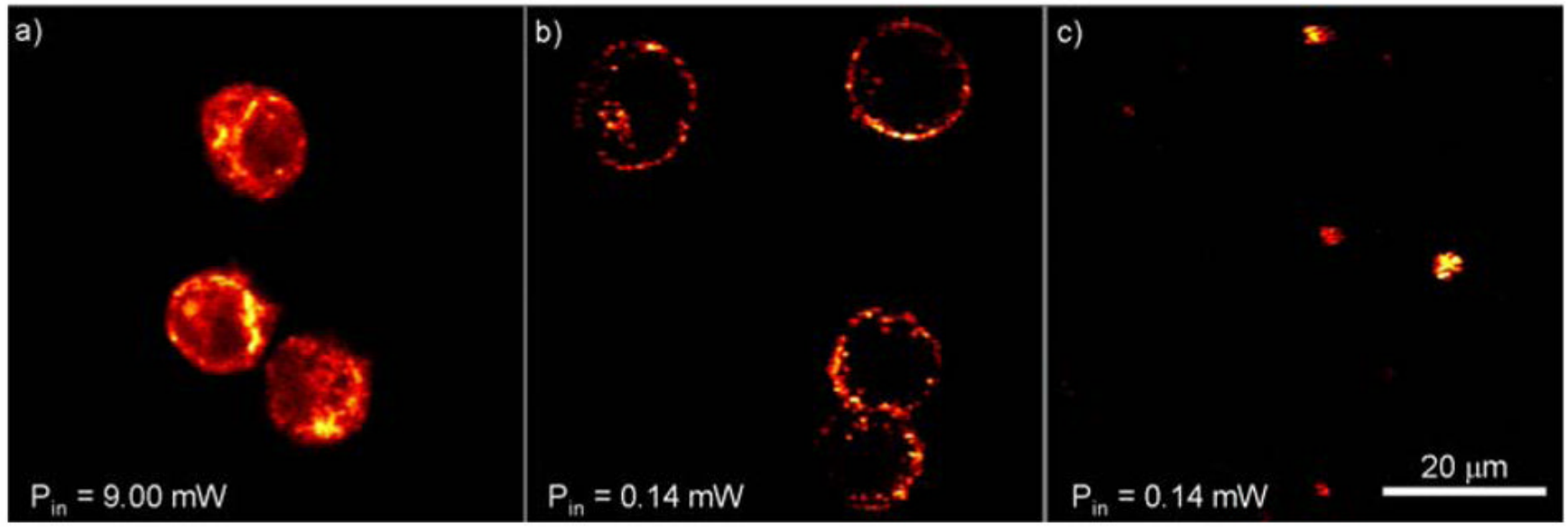

Figure 3.

Two-photon images of cancer cells placed on a coverslip from a cell suspension. (a) TPAF image of unlabeled cells. (b) TPL image of nanorod labeled cells. Imaging required $9 \mathrm{~mW}$ of excitation power in unlabeled cells to get same signal level obtained with only $140 \mu \mathrm{W}$ for nanorod labeled cells, indicating that TPL from nanorods can be more than 4,000 times brighter than TPAF from intrinsic fluorophores. (c) TPL image of non-specifically labeled cells. 
a)

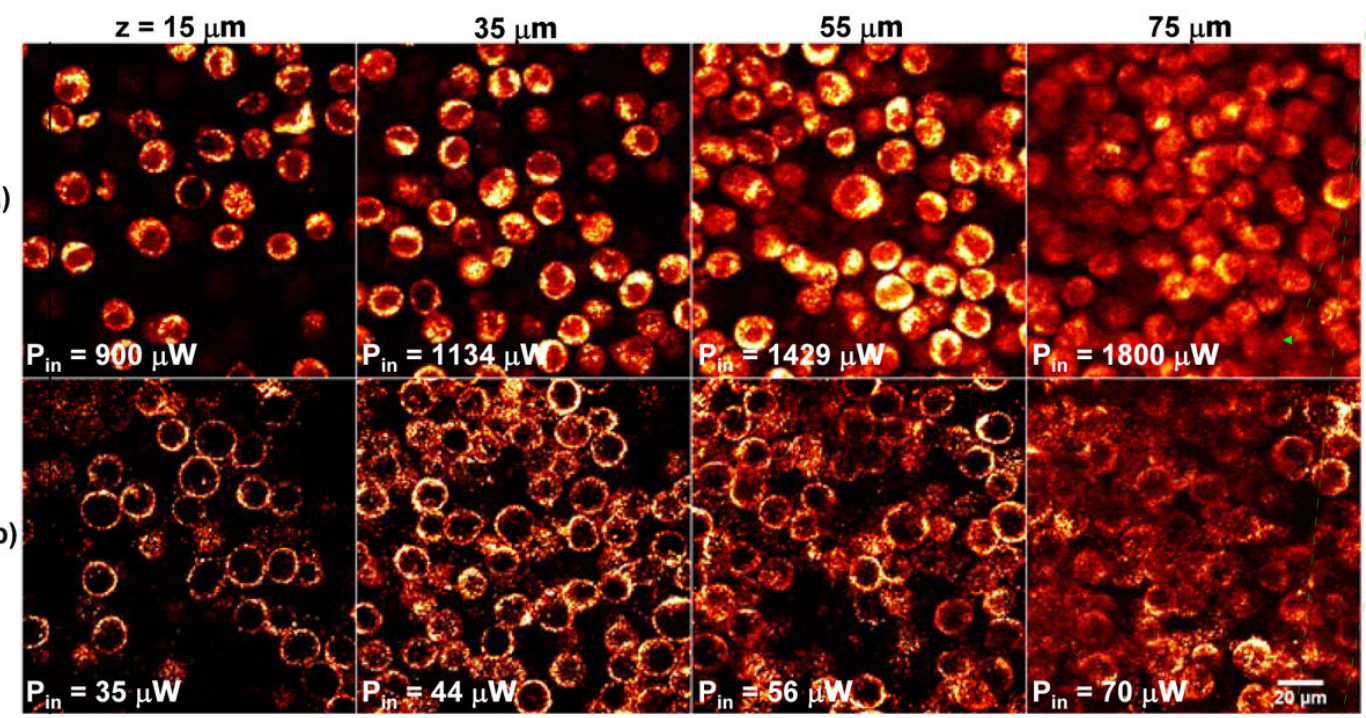

Figure 4.

Two-photon imaging of cancer cells embedded in a collagen matrix at increasing depths. (a) TPAF imaging of unlabeled cells and (b) TPL imaging of nanorods labeled cells. Both samples required the same excitation power increase of $26 \%$ at each $20 \mu \mathrm{m}$ depth increment to maintain constant emission intensity. 